\title{
Diacronie
}

Studi di Storia Contemporanea

$N^{\circ}$ 9, 1 | 2012

Quando la classe operaia andava in paradiso

\section{Intervista a Giuseppe Carlo Marino}

\section{Luca Bufarale e Fausto Pietrancosta}

\section{(2) OpenEdition}

\section{Journals}

\section{Edizione digitale}

URL: http://journals.openedition.org/diacronie/3094

DOI: 10.4000/diacronie.3094

ISSN: 2038-0925

\section{Editore}

Association culturelle Diacronie

Notizia bibliografica digitale

Luca Bufarale e Fausto Pietrancosta, «Intervista a Giuseppe Carlo Marino », Diacronie [Online], № 9,

1 | 2012, documento 16, Messo online il 29 janvier 2012, consultato il 19 avril 2019. URL : http:// journals.openedition.org/diacronie/3094; DOI : 10.4000/diacronie.3094 


\title{
Diacronie
}

\section{6/}

\section{Intervista a Giuseppe Carlo Marino}

\author{
A cura di Luca BUFARALE, Fausto PIETRANCOSTA \\ Redazione del testo dell'intervista a cura di Fausto PIETRANCOSTA
}

Lo storico Giuseppe Carlo Marino affronta alcuni degli aspetti più significativi della storia delle sinistre europee nel secondo dopoguerra. Attraverso una puntuale e accurata analisi del trentennio 1945-1973 viene presentato ed esaminato il ruolo svolto dai partiti e dalle generazioni politiche di sinistra alla prova dei mutamenti e delle trasformazioni politico-culturali e socio-economiche che hanno caratterizzato il secondo Novecento.

Diacronie: Che giudizio dà del periodo definito dalla storiografia francese les trente glorieuses, il trentennio 1945-1973, dal secondo dopoguerra ai prodromi della grande crisi economica degli anni Settanta? Quale analisi si sente di fare sugli aspetti e le caratteristiche che hanno contribuito a definire quegli anni e il rapporto con l'epoca attuale?

Giuseppe Carlo Marino: Trente glorieuses o la più celebre definizione di Eric Hobsbawm "l'età dell'oro"? È lo stesso. Se una siffatta periodizzazione ha la sua base nell'economia (così come la domanda opportunamente suggerisce), occorrerebbe andare indietro fino agli anni Trenta e da lì, in particolare dalla seconda fase del New Deal, procedere in avanti seguendo lo svolgimento di un ciclo unitario delle trasformazioni del capitalismo, segnato dal fordismo e dal keynesismo. Il tutto, pur con assai numerose varianti territoriali (ovvero "nazionali"), sulle linee dinamiche di una società di massa nella quale si sarebbe sempre più intensificata una proficua dialettica produzione-consumo che richiedeva un rigoroso controllo pubblico sulla moneta e sulle attività finanziarie, un'organica composizione (mediante un meccanismo di "concertazione" nei rapporti tra capitale e lavoro) del conflitto sociale con i salari crescenti, nonché l'imposizione di regole e vincoli ad un mercato regolato e razionalizzato dalla politica mediante un'opportuna legislazione. La stesso dramma del 
'29, estesosi dagli Usa all'Europa con effetti assai larghi nel mondo intero, aveva dettato la sua lezione, raccolta dal presidente Franklin Delano Roosevelt dopo il quadriennio fallimentare del suo predecessore Erbert Clark Hoover: il capitalismo avrebbe potuto sopravvivere ad una sorte che sembrava a molti ormai segnata, soltanto trasformandosi in un sistema idoneo a produrre uno sviluppo crescente stimolato dai consumi, a loro volta alimentati da un processo di redistribuzione sociale dei profitti e della ricchezza. In altri termini, un superamento del liberismo e l'impianto di un sistema che, al di là della stessa liberal-democrazia, potrebbe, in senso lato, dirsi "socialdemocratico". La sua concretizzazione in Europa sarebbe avvenuta nei termini di una progressiva affermazione ed estensione dello "Stato sociale" (con esiti persino più avanzati dell'Opulenty Society statunitense, ma sullo sfondo paradigmatico dell'american way of life), a partire dal secondo dopoguerra, una volta travolta la variante autoritarioreazionaria della risposta alla crisi del '29 tentata dai fascismi (che pure, a loro modo va riconosciuto - si erano mossi verso un'atipica forma totalitaria di "Stato sociale"). Dalla parte opposta al capitalismo, si sarebbe svolta, con una forza propulsiva decrescente, la grande sperimentazione del "socialismo reale" nata dalla rivoluzione d'ottobre. Nel complesso, un itinerario unico - seppure in un quadro molto variegato, non uniforme e con molte contraddizioni - segnò quelle epocali trasformazioni del capitalismo che, in Occidente, nell'area euro-atlantica, andarono a sfociare nella prodigiosa stabilizzazione del "trentennio glorioso". Per alcuni anni di quel trentennio i riferimenti "alti” di un'inedita espansione sociale del benessere e della ricchezza sarebbero state la Gran Bretagna laburista e soprattutto le socialdemocrazie nordiche del Continente ${ }^{1}$. Il nostro Paese, del resto come la Germania, fece il suo ingresso più tardi nell'eldorado: nei primi anni Sessanta, dopo la ricostruzione degli anni di De Gasperi e sotto la spinta radicalmente innovatrice del "miracolo econonico"

${ }^{1}$ Cfr. FOURASTIÉ, Jean, Les trentes glorieuses ou la revolution invisible de 1946 à 1975, Paris, Fayard, 1979; CHEVALLIER, François-Xavier, Le Bonheur economique: les trentes glorieuses sont devant nous, Paris, Albin Michel, 1998; HOBSBAWM, Eric J., Il secolo breve 1914-1991, Milano, Rizzoli, 1997 (ediz orig. Age of Extremes. The short Twentieth Century 1914-1991, London, Michael Joseph, 1994); GALBRAITH, Il grande crollo: la crisi economica del 1929, Milano, ETAS, 1966 (ediz. orig. The Great Crash, London, Hamish Hamilton, 1955); FINKELSTEIN, Joseph, The American economy: from the great crash to the third industrial revolution, Arlington Heights, Harlan Davidson, 1992. Si vedano anche BORIONI, Paolo, «La socialdemocrazia nordica e la "sfida democratica al capitalismo"», in Diacronie. Studi di Storia Contemporanea : Quando la classe operaia andava in paradiso, $\mathrm{N}^{\circ} 9,1 \mid 2012$, URL: < http://www.studistorici.com/2012/02/13/borioni_numero_9/ > e STAZZI, Carlo Andrea, «"And Now - Win the peace!". I laburisti inglesi e il Welfare State», in Diacronie. Studi di Storia Contemporanea : Quando la classe operaia andava in paradiso, $\mathrm{N}^{\circ} 9,1 \mid 2012$, URL: < http://www.studistorici.com/2012/o2/13/stazzi_numero_9/ >.

2 Cfr. CRAINZ, Guido, Storia del miracolo italiano, Roma, Donzelli, 1997 (2a ed. ampliata 2003); ID., Il paese mancato. Dal miracolo economico agli anni ottanta, Roma, Donzelli, 2003. 
Personalmente ricordo - ne ho scritto con una certa enfasi nostalgica nel mio Biografia del Sessantotto 3 - che vissi quel senso liberatorio di partecipazione ad una vita di colpo fattasi ottimistica e solare (dopo le plumbee caligini degli anni Cinquanta) ad apertura di quel decennio, mentre si stava aprendo la stagione del "centro-sinistra" 4 : si discuteva, con speranza, di "riforme strutturali", di "programmazione economica", di piena occupazione, di liquidazione della "questione meridionale", di emancipazione femminile, di diritti alla salute, allo studio, alla casa, al salario garantito, nello slancio del "primato" riconosciuto alla politica e nella rivendicazione collettiva dei progressi conseguibili con la socialità, al segno dell' uguaglianza e della giustizia sociale, tra fabbriche in espansione e decine di migliaia di cantieri aperti che stavano rinnovando il volto stesso del Paese. Un tempo favoloso per me. Figuriamoci per i precari e per i giovani di oggi!

Mi si chiede adesso: quale rapporto c'è tra quel passato e i nostri tempi? Non parlerei di un rapporto. Registrerei, piuttosto, una drastica rottura. Se fossi uno dei classici laudatores temporis acti, guardando all'economia e agli attuali diktat del capitalismo finanziario sulla politica, direi di avere a che fare non proprio con una di quelle rotture che innovano la storia, bensì con una sorta di "rottura regressiva" (il ritorno al liberismo e alla conseguenti privatizzazioni in ogni campo, il primato dell'individualismo rampante sulla socialità, la precarizzazione del lavoro, lo smantellamento dello "Stato sociale") che viene capziosamente presentata e perseguita anche nelle forme vaghe e indeterminate, e francamente non poco ingannatrici, del cosiddetto "riformismo"5. Ma non coltivo inclinazioni al rimpianto. E prendo atto del fatto che è cominciata da tempo, almeno a partire dagli anni Ottanta, una nuova fase storica che condurrà le generazioni molto lontano, al di là dei vecchi traguardi della rivoluzione industriale. Solo che, com'è del tutto ovvio, ancora non sappiamo bene dove e con quali effetti per il mondo.

\footnotetext{
3 MARINO, Giuseppe Carlo, Biografia del Sessantotto, Milano, Bompiani, 2004.

4 Ibid., pp. 97-142.

5 Si veda GALLINO, Luciano, (a cura di), Disuguaglianze ed equità in Europa, Roma-Bari, Laterza, 1993; ID., Se tre milioni vi sembran pochi. Sui modi per combattere la disoccupazione in Italia, Torino, Einaudi, 1998; ID., Globalizzazione e disuguaglianze, Roma-Bari, Laterza, 2000; ID., Il costo umano della flessibilità, Roma-Bari, Laterza, 2001; ID., Con i soldi degli altri. Il capitalismo per procura contro l'economia, Torino, Einaudi, 2009; ID., Finanzcapitalismo. La civiltà del denaro in crisi, Torino, Einaudi, 2011.
} 


\section{D.: Cosa ci può dire in generale circa lo stato delle sinistre europee negli anni cinquanta e sessanta nei diversi contesti nazionali? ì possibile innanzitutto fare alcune generalizzazioni? Quali sono a suo avviso le differenze più significative tra le sinistre dei vari paesi?}

G. C. M.: Dovremmo intenderci sul concetto di "sinistra". Il che non è proprio facile. Un punto di possibile accordo su un tracciato argomentativo già indicato da Norberto Bobbio potrebbe essere questo: chi è di sinistra non si accontenta della realtà data, così com'è e si presenta, ma vuole andare oltre: vuole "cambiare" con l'obiettivo di realizzare le condizioni per un avanzamento delle libertà democratiche verso esiti di eguaglianza sociale. È implicita nella vocazione al cambiare per "avanzare" quell'idea di origine settecentesca (che, a pensarci bene, potrebbe essere soltanto utopica) che è l'idea del "progresso" cui si associa quella di giustizia sociale. Ammesso che abbia un senso concreto la parola "progredire", va comunque chiarito se per progresso si intende rendere migliore l'esistente o costruirne un altro diverso, nel senso di opposto e di radicalmente alternativo ${ }^{6}$. Il chiarimento approda, pertanto, ad una netta differenziazione (che è anche un conflitto inevitabile) tra una "sinistra migliorista", detta comunemente socialdemocratica, e una sinistra antisistema, ovvero antagonista, normalmente rappresentata dai partiti comunisti. La guerra fredda avrebbe reso assai drammatica la contrapposizione, essendo le due parti schierate su opposti fronti internazionali, con un riferimento fisso per quella "migliorista" (gli Usa) e con un altro ad apertura variabile (l'Urss, ma anche la Cina e il Terzo mondo) per quella antagonista. A marcare chiaramente la contrapposizione sarebbe stata addirittura la ricostituita Internazionale socialista, organo, piuttosto che di elaborazione strategica, di mera "rappresentanza" di un socialismo che altra prospettiva in concreto non si poneva se non quella di conseguire una gestione più equa e il più possibile "egualitaria" del sistema capitalistico. Per un socialismo di tal genere si era reso quasi naturale un distanziamento dalle fonti marxiste della sua dottrina e della sua elaborazione politica - a partire dal precoce dettato del "revisionismo" già sviluppatosi nella II Internazionale, involutosi nel definitivo abbandono del marxismo del programma di

${ }^{6}$ L'idea di progresso rappresenta uno dei presupposti teorici della modernità e una delle principali basi filosofiche del pensiero moderno. Si può persino intravedere in essa la vera "religione della civiltà occidentale". Storicamente, questa idea si formula intorno al 1680, nel quadro della disputa degli Antichi e dei Moderni, alla quale partecipano Terrasson, Perrault, l'abate di Saint-Pierre e Fontenelle, per poi precisarsi su iniziativa di una seconda generazione, comprendente principalmente Turgot, Condorcet, Louis Sébastien Mercier ed altri, fino ad arrivare al contributo dato dal pensiero di Saint-Simon, Comte, Spencer; Cfr. SALVADORI, Massimo Luigi, L'Idea di progresso. Possiamo farne a meno?, Roma, Donzelli, 2006. 
Bad Godesberg della SPD tedesca (1959; poi Berliner Program del 1989)7 - culminato in una dura contrapposizione con i comunisti. Per i comunisti, i socialdemocratici assestati com'erano sulla frontiera di un cosiddetto "riformismo" (ideologicamente neutro e comunque non marxista se non antimarxista) - ben più che avversari, sarebbero stati addirittura dei nemici: una specie di sindacato giallo al servizio del padronato ovvero della "reazione", come si usava dire. Questo, non soltanto nel periodo tra le due guerre, ma anche molto dopo. Ricordo personalmente (il che può oggi riuscire non poco sorprendente ai giovani e agli stessi membri di formazioni politiche che si dicono di sinistra in quanto vantano progetti "riformisti") che ancora negli anni Settanta-Ottanta del secolo scorso per un comunista dire a un qualsiasi avversario "sei un socialdemocratico" equivaleva a lanciargli contro un'offesa con un misto di irrisione e di disprezzo!

D.: Da un lato la fase espansiva del capitalismo seguita alla ricostruzione postbellica, dall'altro i mutamenti sociali e culturali indotti dall'avvento dei fenomeni caratteristici della società di massa. $E$ ancora: le politiche di welfare, la nazionalizzazione dei settori chiave della produzione, la programmazione economica. Come si sono rapportate le sinistre europee ai processi economici in atto nell'arco del trentennio 1945-1973 e come hanno influenzato le dinamiche sociali che ne sono conseguite?

G. C. M.:La domanda ben periodizza la questione, appunto nell'arco 1945-1973. Infatti, dopo - con i primi segnali di "sofferenza" del sistema neocapitalistico nato dalle riforme keynesiane, poi cresciuti fino a rendere diffuso nelle stesse socialdemocrazie più strutturate e avanzate del Nord Europa l'allarme per l'insostenibilità dello "Stato sociale" (la "crisi fiscale dello Stato" denunziata da James O’Connor) - si sarebbe evidenziata un po' ovunque, in Europa, la crisi delle politiche di welfare. In quel trentennio, erano state soprattutto le sinistre (quelle "miglioriste" o socialdemocratiche che i comunisti stigmatizzavano in quanto e perché funzionali alla salvaguardia e allo sviluppo del capitalismo) le protagoniste della grande trasformazione che aveva fatto della società di massa la "società dei consumi". Soprattutto in Svezia e in Danimarca,

\footnotetext{
7 Bad Godesberg è la cittadina tedesca vicino Bonn in cui dal 13 al 15 novembre 1959 la socialdemocrazia tedesca celebrò il congresso della "svolta". Cfr. COLOTTI, Enzo, La Socialdemocrazia tedesca, Torino, Einaudi, 1959 e TRALDI, Francesca, Verso Bad Godesberg: la socialdemocrazia e le scienze sociali di fronte alla nuova società tedesca (1945-1963), Bologna, Il Mulino, 2010.
} 
ma anche in Germania con la SPD, ben più che "influenzare le dinamiche sociali", sono state determinanti: quelle dinamiche sociali le hanno tout court lanciate e guidate nel loro svolgimento, favorendo una distribuzione sociale della ricchezza collettiva nella forma di una piuttosto larga ed evidente "socializzazione dei profitti”. Il che, come ben sappiano (e come rilevavano, già allora, da opposti punti di vista, sia le sinistre antagoniste, sia le destre liberali) non senza produrre o accentuare altre contraddizioni. Per le sinistre antagoniste, quel processo non rimuoveva affatto le radici delle ingiustizie sociali e soltanto ne mistificava la "gestione strumentale" da parte del sistema capitalistico alienando la coscienza di classe del proletariato, subdolamente acquietando il conflitto sociale nella gara per il "benessere" e nel conseguente consumismo funzionale ai profitti, sì da fare dell' Europa occidentale un'area di privilegio neoimperialistico (in associazione subalterna con gli Usa), costruita sullo sfruttamento dei tre quarti del pianeta che ne restava ai margini o esclusa; per le destre liberali, di contro, si trattava di una dissennata affermazione dello statalismo ai danni dell'iniziativa privata e delle libertà del mercato di cui lo Stato stesso avrebbe pagato presto le conseguenze in termini di un insostenibile indebitamento pubblico e la società in termini di inflazione, di disinteresse per il lavoro produttivo e di progressivo esaurimento delle capacità di ulteriore crescita economica (tutto il repertorio delle critiche avanzate dai liberisti della Scuola di Chicago). Scontato che sia la sinistra antagonista che le destre liberali avessero, come avevano, dei buoni argomenti ai quali affidarsi per avanzare le loro critiche, resta il fatto che le politiche socialdemocratiche determinarono un vero e proprio salto di qualità nel sistema capitalistico di cui per lungo tempo le masse popolari (soprattutto in virtù del full-employment e dei salari crescenti) avrebbero avvertito più $i$ vantaggi immediati che le persistenti contraddizioni, nonché gli effetti perversi e i limiti di prospettiva. Di quest'ultimi (gli effetti perversi e i limiti di prospettiva) le sinistre europee, sia quelle socialdemocratiche che quelle antagonistiche, si sarebbero accorte con non poco ritardo per quel che riguarda le conseguenze dello sviluppo sull'ambiente naturale della vita organizzata: questioni, poi, sollevate con crescente allarme, dai Verdi.

Nel contempo, con un crescendo successiva agli anni Ottanta, la sinistra socialdemocratica avrebbe vissuto la lunga ed irreversibile agonia del suo progetto all'interno del sistema, a causa della "crisi fiscale" denunziata e studiata da O'Connor: sarebbe, infatti, venuta meno la possibilità di operare, tramite lo Stato, per la redistribuzione dei profitti ${ }^{8}$.

8 O'CONNOR, James, La crisi dello Stato fiscale, Torino, Einaudi, 1977. 
D.: In che modo la coscienza antifascista e la consapevolezza delle conquiste democratiche raggiunte hanno condizionato l'azione delle sinistre europee?

G. C. M.: In un modo determinante, almeno fino al Sessantotto. Essere di sinistra ed essere antifascisti era la stessa cosa, sia per i "miglioristi” che per gli antagonisti. Il che, ovviamente, non significa affatto che poi le due parti avessero modalità similari e concordi per dirsi "democratiche" o "socialiste". Il più forte legame con l'antifascismo (un legame, che direi quasi costitutivo della loro stessa ragione storico-politica) lo si registra per il PCF e per il PCI9. E, certo, non soltanto in conseguenza del ruolo decisivo svolto dall'Urss e dall'Armata rossa nella guerra contro il nazifascismo. Ma per i compiti di cui quei due partiti si erano ben più degli altri investiti nelle rispettive Resistenze nazionali. Si pensi alla Francia: lì la bandiera della Resistenza fu inalberata da De Gaulle, ma senza i comunisti il generale non avrebbe fatto molta strada e non avrebbe poi potuto vantare alcun successo. E, in Italia, senza i comunisti non sarebbe mai nata una Resistenza degna del suo nome e non si sarebbe mai sviluppata una vittoriosa guerra di liberazione.

D.: L'integrazione europea e la tensione tra i blocchi, sono stati due nodi che hanno costituito un'occasione di confronto e di scontro tra le varie anime della sinistra. Quali punti di contatto o di differenziazione hanno prodotto il processo di costruzione della Comunità Europea e la contestuale rilettura della recente storia del continente sui partiti, sulle classi dirigenti e sull'opinione pubblica di sinistra in Europa?

G. C. M.:Al confronto e ai motivi di scontro, riferibili anche al quadro della guerra fredda, ho già accennato. Per quanto riguarda il processo di integrazione europea, era scontato che la sinistra antagonistica vi scorgesse il pericolo di una specie di "normalizzazione geopolitica" a consolidamento, in Europa, dell'“impero americano" nell'orbita atlantista della Nato. Non a caso i comunisti francesi (pur con tutto il loro noto, tenacissimo legame con l'Urss e il "socialismo reale") furono per molti versi, e paradossalmente, in sintonia con il generale De Gaulle che della Nato $e$ dell'“impero americano" (nonché di una certa Europa) era un convinto avversario. Tuttavia, anche

9 Cfr. MARTIGNONI, Martina, «Due vie democratiche al socialismo? PCI, PCF e il concetto di nazione tra il 1944 e il 1947», in Diacronie. Studi di Storia Contemporanea : Quando la classe operaia andava in paradiso, $\mathrm{N}^{\circ} 9,1 \mid 2012$,

URL: < http://www.studistorici.com/2012/02/13/martignoni_numero_9/ >. 
se è vero che una parte rilevante nell'attivazione dei processi di integrazione europea spettò principalmente al ceto politico del cattolicesimo democratico, tutte le sinistre (compresa quelle comuniste) entrarono in tempi diversi nell'orbita delle iniziative concrete per l'unificazione europea su un filo comune costituito dalla tradizione pacifista e internazionalista del movimento operaio: un filo - al quale si annodavano, in Italia, anche quelli della tradizione mazziniana (si pensi in particolare, al precoce orientamento europeista della sinistra rosselliana e azionista) - che, avvolgendo in vario grado tutte le forme organizzate di coscienza democratica, non poteva non coinvolgere, a maggior ragione, tutti i più vari richiami al socialismo ${ }^{10}$. Con la costruzione dell'Europa era in gioco, per le socialdemocrazie, sia la fine definitiva del cosiddetto "pericolo tedesco" per la pace, sia l'avvenire di una pacifica espansione e condivisione dei sempre più incerti vantaggi offerti alle masse dal "capitalismo sociale". Mentre, poi, nell'inedita, assai originale costruzione ideale dell'eurocomunismo di Enrico Berlinguer ${ }^{11}$, l'europeismo sarebbe stato indicato come strada maestra per realizzare una “democrazia reale" (e quindi un'autentica società socialista) al di là del capitalismo e della subalternità al capitalismo dei partiti socialdemocratici. Naturalmente, sugli orientamenti berlingueriani influiva molto la peculiare lezione di Gramsci sulle esigenze tattico-strategiche della cosiddetta "guerra di posizione" come fase preliminare e necessaria per il cambiamento rivoluzionario in aree di capitalismo maturo quali erano certamente quelle dell'Europa occidentale ${ }^{12}$.

10 Sull'orientamento europeista della sinistra rosselliana e azionista cfr. MALANDRINO, Corrado, Socialismo e libertà. Autonomie, federalismo, Europa da Rosselli a Silone, Milano, Franco Angeli, 1990; GRAGLIA, Piero, Unità europea e federalismo. Da «Giustizia e Libertà» ad Altiero Spinelli, Bologna, Il Mulino, 1996. Si vedano ADESSO, Serena, «Il consenso delle sinistre italiane al processo di integrazione europea (1950-1969)», in Diacronie. Studi di Storia Contemporanea : Quando la classe operaia andava in paradiso, $\mathrm{N}^{\circ}$ 9, 1|2012, URL: < http://www.studistorici.com/2012/02/13/adesso_numero_9/ > e COLOZZA, Roberto, «Guardare lontano. Modelli, esplorazioni e collaborazioni internazionali del movimento di Unità Popolare (1953-1957)», in Diacronie. Studi di Storia Contemporanea : Quando la classe operaia andava in paradiso, $\mathrm{N}^{\circ} 9,1 \mid 2012$,

URL: < http://www.studistorici.com/2012/02/13/colozza_numero_9/ >

${ }^{11}$ POZZOLI, Bona, RENZI, Emilio (a cura di), Eurosocialismo ed eurocomunismo. Lineamenti cronologici e bibliografici, Milano, Feltrinelli, 1977; BIANCO, Gino (a cura di), Eurocomunismo e socialismo democratico, Pistoia-Roma, Fondazione Turati, 1978; VALLI, Bernando, Gli eurocomunisti, Bompiani, Milano 1977; FERRARI, Paolo, In cammino verso Occidente. Berlinguer, il PCI e la comunità europea negli anni '7o, Bologna, Clueb, 2007; DI GIACOMO, Michelangela, «Identità eurocomunista: la traiettoria del Pce negli anni Settanta», in Studi Storici, 2, 2010, pp. 461-492.

${ }_{12}$ Cfr. MARINO, Giuseppe Carlo, Autoritratto del Pci staliniano 1946-1953, Roma, Editori Riuniti, 1991. 


\section{D.: Quale influenza hanno avuto i movimenti anticolonialisti e le} rivoluzioni in Asia, Africa e America Latina (rivoluzione cinese, rivoluzione cubana, guerra del Viet Nam, lotte di liberazione nelle colonie portoghesi etc.) nel dibattito all'interno delle sinistre europee e nella ridefinizione del concetto di imperialismo? A suo avviso la seconda metà degli anni settanta, con la crisi dei movimenti di liberazione e la loro degenerazione in regimi burocratico-autoritari, ha costituito una frattura importante nella visione che le sinistre europee avevano del "Terzo Mondo" e delle sue possibilità di emancipazione?

G. C. M.: Un'influenza assai rilevante nel dibattito della sinistre di formazione e tradizione comuniste. Addirittura, in tale orbita, provocarono, negli anni sessanta (con esiti destinati ad approfondirsi negli anni successivi), una frattura che, in Italia, fu quella tra "Potere operaio" e "Lotta Continua"13, determinata dal contrapposto giudizio sulla natura del potere sovietico, sulla funzione internazionale dell'Urss e persino su Lenin e sul leninismo. Per "Lotta Continua" (ed anche per il gruppo de «il manifesto») tornò in voga, tra gli altri, Leone Trockji, riesumato dalla sua tragica vicenda di eresiarca e assunto tra i riferimenti alti e "nobili" del comunismo antistalinista. Essere comunisti guardando all'Urss - ovvero alle sue trasformazioni dopo la rivoluzione di ottobre per effetto dello stalinismo e poi del neostalinismo brezneviano ${ }^{14}$ - significò

13 Cfr. VETTORI, Giuseppe (a cura di), La sinistra extraparlamentare in Italia. Storia. Documenti. Analisi politica, Roma, Newton \& Compton, 1973; VALLAURI, Carlo, I gruppi extraparlamentari di sinistra. Genesi e organizzazione, Roma, Bulzoni, 1976; MONICELLI, Mino, L'ultrasinistra in Italia 1968-1978, Roma-Bari, Laterza, 1978; GIACHETTI, Diego, I partiti della nuova sinistra: origini, sviluppo, epilogo, in BILLI, Fabrizio (a cura di), Gli anni della rivolta. Prima, durante e dopo il '68, Milano, Punto Rosso, 2001, pp. 85-102. Cfr. anche PERRI, Paolo, «La nuova sinistra e i nazionalismi periferici» in Diacronie. Studi di Storia Contemporanea : Quando la classe operaia andava in paradiso, $\mathrm{N}^{\circ} 9,1 \mid 2012$, URL: < http://www.studistorici.com/2012/o2/13/perri_numero_9/ > e SACCHETTI, Giorgio, «Eretici e libertari. Il movimento anarchico in Italia (1945-1973)», in Diacronie. Studi di Storia Contemporanea : Quando la classe operaia andava in paradiso, $\mathrm{N}^{\circ} 9,1 \mid 2012$, URL: < http://www.studistorici.com/2012/02/13/sacchetti_numero_9/ >.

${ }_{14}$ Un ruolo rilevante nella rivalutazione del pensiero di Trockij e di altre figure "eretiche" del comunismo fu assunto all'epoca dalla casa editrice romana Samonà e Savelli che pubblicò ad esempio il testo "In difesa del marxismo", una raccolta di interventi e lettere di Trockij dallo scoppio della seconda guerra mondiale al suo assassinio un anno dopo. Cfr. TROCKIJ, Lev Davidovič, In difesa del marxismo, Roma, Samonà e Savelli, 1969. Tra gli intellettuali marxisti vicini alla "nuova sinistra" che più si spesero all'epoca per la rivalutazione del pensiero del rivoluzionario russo in chiave antistalinista vi è Sebastiano Timpanaro. Cfr. TIMPANARO, Sebastiano, BELGRADO, Franco, «Quel "cane morto di Lev Davidovič», in Giovane critica, 30, 1972, pp. 56-59, ora anche in TIMPANARO, Sebastiano, Il verde e il rosso. Scritti militanti 1966-200o, Roma, Odradek, 2001, pp. 41-49. Cfr. anche AGA-ROSSI, Elena, ZASLAVSKY, Victor, Togliatti e Stalin. Il PCI e la politica estera staliniana negli archivi di Mosca, Bologna, Il Mulino, 1997; AVTORCHANOV, Abdurachman, La tecnologia del potere. Il potere nell'URSS da Stalin a Breznev, Milano, La casa di Matriona, 1980. 
altra cosa dall'essere comunisti guardando alla rivoluzione cinese (in ispecie a quella "culturale" di Mao Zedong di cui si ignoravano le sanguinose violenze) e alla Cuba guevarista e al Vietnam di Ho Chi Minh . Si era formato, in un certo senso, un "neocomunismo", una sorta di "comunismo libertario" - del tipo di quello che in Italia ebbe la sua migliore espressione appunto nel gruppo de «il manifesto» - parallelo e simmetrico al cosiddetto neoimperialismo nel quale, ormai, i "neocomunisti" includevano la stessa Unione Sovietica: un'inclusione, mi sembra, non proprio corretta, dato che la politica internazionale dell'Urss, pur con mille contraddizioni, tramite la stessa espansione della sua egemonia sui movimenti di liberazione e sui nuovi assetti geopolitici che ne derivavano, mirava a disarticolare il quadro mondiale dell'imperialismo americano appoggiando (come nell'Egitto di Nasser) le lotte guidate dalle borghesie nazionali. Naturalmente, poi, la deriva burocratico-autoritaria di alcuni movimenti di liberazione nel loro farsi potere statuale (penso, oltre che agli sviluppi della situazione politica nel Vietnam liberato, alla stessa Cuba di Fidel Castro, ma anche alla realtà africana nella quale, a parte la sempre controversa Libia di Gheddafi, non c'era molto da sperare dalla Tunisia di Habib Bourguiba, dall'Egitto postnasseriano di Anwar Sadat, dalla Somalia di Mohammed Barre e dall'Etiopia di Haile Mengistu) generò disorientamento, nonché molte travagliate sospensioni del giudizio e, più in generale, delusione. Non era conforme alle speranze delle sinistre antagoniste (sempre più costituite da litigiose formazioni "neocomuniste" più o meno antisovietiche) il dover prendere atto del fatto che le cosiddette "rivoluzioni nazionali" dei paesi del Terzo mondo ${ }^{15}$ non avevano alla loro base un alcunché di "proletario" in senso proprio marxiano, ma erano tutt'al più degli slanci anticolonialisti guidati da "ribelli" ed élite locali (spesso di origine militare) la cui vocazione progressista era molto dubbia o comunque fortemente limitata da condizioni strutturali di arretratezza. È ovvio che della delusione stessero in vario modo approfittando le sinistre "miglioriste" nel tentativo di reagire al loro tramonto riproponendosi come una "terza via" tra capitalismo e "socialismo reale (penso alla SPD di Willy Brandt, ma soprattutto a quel singolare socialismo liberista rappresentato da Tony Blair).

\footnotetext{
15 Per una originale critica marxista delle "rivoluzioni nazionali" dei paesi del Terzo mondo cfr. GRANT, Ted, La rivoluzione coloniale e gli stati operai deformati, in ID., Il lungo filo rosso. Scritti scelti 1942-2002, Milano, A. C. Editoriale Coop, 2007 (l'articolo è risalente al luglio 1978). Cfr. PERRI, Paolo, «La nuova sinistra e i nazionalismi periferici» in Diacronie. Studi di Storia Contemporanea : Quando la classe operaia andava in paradiso, $\mathrm{N}^{\circ}$ 9, 1|2012, URL: < http://www.studistorici.com/2012/02/13/perri_numero_9/ > .
} 


\section{D.: In che modo i partiti della sinistra si sono rapportati ai movimenti collettivi della fine degli anni Sessanta? Quali relazioni virtuose, criticità o distanze sono nate e si sono consolidate tra la sinistra "istituzionale" e questi movimenti?}

G. C. M.: Invero, parlare di "sinistra istituzionale" non è una forzatura terminologica soltanto se - pur salvaguardandosi l'idea fondamentale della sinistra che è quella, come ho già detto, di "cambiare" la realtà in funzione del "progresso" - tale opposizione si trasferisce nelle forme istituzionali di un sistema rappresentativo di tipo liberaldemocratico (la formazione dei ceti dirigenti tramite i partiti e i sindacati, le procedure elettorali e quelle, soprattutto, per la produzione legislativa tramite il parlamento) sì da diventare, laddove non riesca a divenire essa stessa "governo" o a parteciparvi, qualcosa di simile, per dirla alla maniera degli inglesi, a un'“opposizione di Sua Maestà". Questa condizione riguarda ogni tipo di "sinistra migliorista”, o riformista o socialdemocratica che dir si voglia. Mi sembra scontato che quanti coltivino un'idea di sinistra come opposizione al sistema non possano che trovarsi da un'altra parte rispetto alla cosiddetta "sinistra istituzionale". Di relazioni "virtuose" tra i due fronti non riuscirei ad indicarne. Semmai, insisterei sulle distanze, molto rilevanti, fino allo scontro, soprattutto laddove, come in Italia, il conflitto sociale è stato molto intenso e talvolta drammatico: uno scontro che la componente più estremista ha spinto talvolta, come è noto, fino alla "lotta armata", senza escludere il terrorismo. La "sinistra istituzionale" è stata accusata dall'altra, l'“antagonista”, di essere funzionale agli interessi del padronato e di rappresentare, nel migliore dei casi, un'opposizione di facciata. Una siffatta critica, da parte dei movimenti (assumendo una configurazione detta, dal punto di vista delle istituzioni, "extraparlamentare"), ha investito in tempi piuttosto recenti, come è noto, anche il Pci e gli stessi sindacati confederali (si ricordi l'attacco dei cosiddetti "autonomi” alla Cgil di Luciano Lama ${ }^{16}$ ). Di qui la storia di una divaricazione a sinistra che per me costituisce appena un passato prossimo , mentre, per i giovani, è già un passato remoto.

Il massimo della divaricazione si evidenziò quando il Pci, da opposizione parlamentare (e sempre meno opposizione sociale), divenne addirittura governo con la Dc di Andreotti, ai tempi della "solidarietà nazionale". Allora, i movimenti “extraparlamentari”, nati, alla fine degli anni Sessanta e ulteriormente sviluppatisi con la loro esuberante combattività nel decennio successivo, divennero il bacino di

${ }^{16}$ Cfr. TURONE, Sergio, Storia del sindacato in Italia 1943/1980, Roma-Bari, Laterza, 1973; ALOSCO, Antonio, Alle radici del sindacalismo. La ricostruzione della Cgil nell'Italia liberata, Milano, Sugarco, 1979. 
un'opposizione antagonistica di tale intensità e drammaticità da rendere improponibile l'ipotesi che tra essi e la "sinistra istituzionale" (compreso il Pci) potesse intercorrere una qualsiasi "relazione virtuosa". D'altra parte, mi chiedo e chiedo, che cosa è da intendersi per "virtuoso"? Non mi sembra che sia corretto applicare una categoria di giudizio morale ai processi storico-concreti della lotta politica. E, anche se fosse corretto, non saprei proprio come farlo.

\section{D.: Come la crisi economica degli anni Settanta ha contribuito a ridefinire il panorama politico delle sinistre in Europa?}

G. C. M.: Forse è ancora troppo presto per tentare un'analisi accurata di quanto è accaduto dagli anni Settanta in poi. E risulta parecchio difficile persino aver chiari e concreti riferimenti per identificare oggi la "sinistra" in una convulsione di cambiamenti epocali che sta rendendo almeno antiquate se non addirittura del tutto inservibili le categorie in uso nella cultura politica dell'età della "rivoluzione industriale“ di cui la cosiddetta "postmodernità" - ovvero la nuova rivoluzione strutturale postfordista che già nel 2000 ho definito, nel mio quasi ignorato Eclissi del principe e crisi della storia, "elettronico-informatica" - sta evidentemente segnando la fine, con la progressiva de-industrializzazione e con le conseguenti radicali trasformazioni in fieri nella morfologia delle classi e dei ceti sociali che stanno mettendo in liquidazione sia la tradizionale "classe operaia", sia la stessa tradizionale "borghesia"17. Ecco, piuttosto che di "crisi economica" - ma quanto è lunga, di grazia, questa crisi! - parlerei di una "svolta epocale" consistente nell'apertura di un nuovo corso storico, di per sé rivoluzionario (nel senso dei processi rivoluzionari indicati da Marx ed Engels per lo sviluppo storico dei "sistemi di produzione"), di cui non è ancora possibile prevedere le forme mature di stabilizzazione nell'economia e nella società. Del resto - l'ho già scritto altrove e lo vado ripetendo - avrebbero mai potuto prevedere i deputati degli Stati generali francesi dove sarebbe arrivato alla fine il processo rivoluzionario di cui essi erano gli ancora inconsapevoli protagonisti all'atto del "giuramento della Pallacorda"? Certo non pare che esista ai nostri giorni una qualsiasi sala della pallacorda nella quale "giurare", da sinistra, per cambiare la realtà di un mondo che sempre più produce insicurezza e angoscia. Prevale, con l'insicurezza, la tendenza alla frantumazione. Un po' tutti restano confusi e si dividono dinanzi alle politiche e alle economie neoliberiste; e i "miglioristi" (riformisti), in particolare,

${ }_{17}$ MARINO, Giuseppe Carlo, Eclissi del principe e crisi della storia. Apogeo e tramonto della democrazia rivoluzionaria nel XX secolo, Milano, Franco Angeli, 2000. 
sembrano rassegnati a diventarne sostanzialmente i complici sofferenti, subendo il diktat della globalizzazione capitalistica ${ }^{18}$. La crisi politica delle sinistre è evidenziata dai risultati delle consultazioni elettorali svoltesi nel 2010 in diversi Stati europei: i socialisti francesi crollano ad un misero 16\%; i tedeschi al 21\%, gli svedesi al 24\%; vanno meglio, ma in discesa, i socialisti portoghesi di José Socrates che perdono la maggioranza assoluta. Eppure il SE (il partito della Sinistra Europea) potrebbe ancora contare su forze ancora assai rilevanti nei vari Paesi: in Germania il 21-23\% dell'elettorato sul quale per adesso riesce ad attestarsi la SPD, se unito a quello di una LINKE al 12\% e all'altro dei Verdi al 10\%, oscillerebbe tra il 48 e il 50\%; in Portogallo, sommando il $36 \%$ dei socialisti di Socrates agli altri della sinistra (il 10\% dell'altra formazione socialista, l'8\% di comunisti e verdi) si otterrebbe un rassicurante $54 \%$. Superando la frantumazione, analoghi risultati potrebbero realizzarsi nell'immediato per la sinistra greca di George Papandreou e, in prospettiva, anche in Francia, in Spagna e in Italia. Solo che, in concreto, la divaricazione tra "miglioristi” e antagonisti non solo impedisce la formazione di un fronte comune, ma riaccende il fuoco di vecchi, insuperati contrasti sulla questione degli orientamenti strategici nei confronti del capitalismo. In questo, sembra sempre più che i "miglioristi", ovvero i cosiddetti "riformisti", al di là dei proclami di opposizione, si stiano addirittura adattando a ritenere ineluttabile il corso liberista del capitalismo funzionale alla globalizzazione: un'evidente, grave involuzione del tradizionale orientamento keynesiano delle socialdemocrazie.

\section{D.: Si può ritenere corretta una lettura che vede l'uso del termine "sinistra"} non solo nell'accezione partitica ma soprattutto come espressione dell'incontro tra culture politiche, orientamenti di pensiero e visioni del mondo, spesso anche molto differenti tra loro ? Che ruolo ebbero in tal senso le realtà non propriamente partitiche nella ridefinizione della sinistra?

G. C. M.: Il termine "sinistra" non è mai stato usabile soltanto nell'accezione partitica. Non si è di sinistra (e,simmetricamente, non si è di destra) senza una specifica, seppure molto larga e sempre dilatabile, "visione del mondo" che nasce dal confronto dialettico tra diversi fuochi di elaborazione e di riflessione sulla condizione umana alla ricerca di un qualche senso da dare alla vita (individuale e sociale), nonché, complessivamente

${ }_{18}$ Cfr. BAUMAN, Zygmunt, Dentro la globalizzazione. Le conseguenze sulle persone, RomaBari, Laterza, 1998. 
alla storia. Il marxismo non sarebbe nato senza Hegel e l'hegelismo, senza la dialettica tra illuminismo e filosofia romantica, senza il confronto, sui grandi temi delle libertà reali e dei diritti del lavoro a fronte delle ingiustizie e delle oppressioni; una dialettica che aveva già attraversato sia le correnti filantropiche laiche sia altre di origine cristiana, nonché il dibattito dei socialisti premarxiani. Guardando ai processi più vicini a noi nel tempo, appare evidente che alla formazione di una cultura politica di sinistra, ben al di là dei partiti e spesso a prescindere dalle loro specifiche vicende, abbiano grandemente contribuito certe importanti culture politiche "trasversali" e radicali quali quelle scaturite dalla riflessione sui diritti umani e sui diritti civili, sulla condizione femminile (il femminismo), sulla scienza e sulla natura per la preservazione dell'ambiente della vita (l'ecologismo), ecc. E hanno anche contribuito le visioni utopiche e profetiche (compresa quella cattolica che in Italia aveva già conosciuto l'originale elaborazione cattolico-comunista di Felice Balbo e di Franco Rodano). Per questo amo ripetere che, a maggior ragione in un'età come la nostra - che rende assai ardua la ricostituzione di un'identità della sinistra dinanzi al permeante "pensiero unico" della globalizzazione capitalistica - è importante, addirittura urgente, che i giovani riattivino la dialettica tra la realtà e l'utopia (si veda, in proposito il mio recente Globalmafia). Mi sembra che qualcosa del genere stia già accadendo con il movimento degli indignados. Altra cosa sarà mettere a punto un'efficace strategia ${ }^{19}$.

\section{D.: Quale ruolo hanno svolto le diverse generazioni politiche nella costruzione dell'identità della sinistra?}

G. C. M.: Le generazioni - un concetto, questo di "generazione", assai complesso e difficilmente usabile in storiografia, anche se io, come qualcuno sa, ho provato ad usarlo- all'atto della loro epifania giovanile hanno sempre avuto la tendenza a produrre opposizione al sistema, ma nell'orizzonte dei "valori" prodotti e professati dalle componenti progressiste della tradizione culturale e politica, ovvero della "paternità sociale", con la quale sono solite confrontarsi allinterno del medesimo sistema: in altri

\footnotetext{
19 MARINO, Giuseppe Carlo, Globalmafia. Manifesto per uninternazionale antimafia, Milano, Bompiani, 2011; BASSI, Jacopo, PIETRANCOSTA, Fausto, «Intervista a Giuseppe Carlo Marino Le mafie globali e l'internazionale antimafia», in Diacronie. Studi di Storia Contemporanea: Storia transnazionale e prospettive transnazionali nell'analisi storica, $\mathrm{N}^{\circ} 6,2 \mid 2011$, URL: $\quad<$ http://www.studistorici.com/2011/04/29/bassi-pietrancosta_numero_6/>; PIETRANCOSTA, Fausto, «Giuseppe Carlo MARINO, Globalmafia. Manifesto per un'Internazionale antimafia», in Diacronie. Studi di Storia Contemporanea : Storia transnazionale e prospettive transnazionali nell'analisi storica, $\mathrm{N}^{\circ} 6,2 \mid 2011$, URL: < http://www.studistorici.com/2011/04/29/pietrancosta_numero_6/>.
} 
termini, tendono a contestare ai "padri" di non essere stati all'altezza dei loro propositi , se non, addirittura, di avere eluso o falsificato o, peggio ancora, tradito, i valori costitutivi delle loro profferte di modernità e di progresso civile. Si sono mostrate inclini a criticare e a superre il presente assumendo come riferimento il passato. Così come accadde nel '68 quando in Germania i giovani contestavano ai padri di essere rimasti sostanzialmente dei nazisti mentre esibivano un ricostituito volto democratico e, in Italia, quando i giovani accusavano i padri di avere eluso o accantonato il portato ideale della Resistenza e dell'antifascismo. Ma, dopo il '68, qualcosa si è rotto in questa dinamica: le generazioni emergenti, soprattutto dagli anni Ottanta in poi, quasi certamente per effetto dei cambiamenti epocali indotti dal passaggio alla "postmodernità", hanno preso a guardare quasi esclusivamente al futuro ${ }^{20}$. Si tratta, mi sembra, di un integrale processo di de-storicizzazione della "visione del mondo", della cultura, della progettualità sociale e della politica e delle stesse istanze di cambiamento del presente (percepito come sempre più evanescente e funzionale al futuro), che sta investendo anche la sinistra. La corsa al futuro va rendendo sempre meno consapevoli dell'esigenza di prepararlo, di fondarlo questo - assai spesso adulato - futuro, confrontando il presente con il passato. La storia viene così sepolta nei suoi archivi, al massimo delegata a certi antiquati specialisti della memoria il cui sapere sarebbe inutile per la costruzione dell'avvenire; una "crisi della storia" ${ }^{21}$ forse irreversibile ovvero, per meglio dire, della "storicità", appunto come ho già scritto in un saggio appena citato pocanzi che pochi hanno avuto la pazienza di leggere. Se ne producono effetti di distorsione e di falsificazione (spesso del tutto inconsapevoli) sul concetto e sullo stesso linguaggio politico di quanti si dicono ancora di sinistra. Il che è da rilevare ampiamente per le parole "riformismo" e "riformista" che spesso si pronunciano, direi ormai quasi normalmente, senza avere idee chiare su quel che le rende di per sé incompatibili con le istanze - pur ancora talvolta confusamente avvertite e intenzionalmente coltivate - di un reale, profondo, strutturale cambiamento della realtà.

${ }^{20}$ Cfr. MARINO, Giuseppe Carlo, Le Generazioni italiane dall'Unità alla Repubblica, Milano, Bompiani, 2006.

${ }^{21}$ Cfr. MARINO, Giuseppe Carlo, Eclissi del principe e crisi della storia. Apogeo e tramonto della democrazia rivoluzionaria nel $X X$ secolo, cit. 
D.: Socialdemocratici, laburisti e socialisti, comunisti ortodossi e dissidenti, anarchici e trotskisti: è condivisibile la lettura in base alla quale tra queste anime e questi gruppi della sinistra contaminazioni e osmosi furono più frequenti di quanto normalmente si ritenga? La classica distinzione tra "riformisti" e "rivoluzionari" può sempre essere adottata in maniera univoca e monolitica o vanno proposte distinzioni che configurino diversi e a volta paralleli percorsi delle varie realtà della sinistra in Europa in base al paese e al momento storico?

G. C. M.: Certamente le differenze di strategia e di "vissuto sociale" di quel che chiamasi "sinistra" sono state rilevanti, da Paese a Paese, in rapporto ai diversi gradi di intensità e di estensione del conflitto sociale in ciascuna realtà e in rapporto alle rispettive tradizioni "nazionali" dei movimenti di massa con un particolare ruolo di quelle costituite storicamente dai movimenti operai. E, naturalmente, le differenze non escludono né i confronti dialettici, né le osmosi e le reciproche contaminazioni. Si pensi alla netta differenza tra il laburismo britannico nato e sviluppatosi su un itinerario di originaria e reiterata autonomia dal marxismo rispetto alle altre dell'Europa continentale per le quali il rapporto con il marxismo è stato invece costitutivo, almeno dal 1848 in poi. Ma è anche vero che la stessa storia del laburismo ha generato, al suo interno, delle componenti "marxiste" che sono ancora presenti come correnti "comuniste" - per quanto assai minoritarie - nello stesso Labour Party ${ }^{22}$. Anche per tutte le altre "differenze" (spesso, molto più che "differenze", vere e proprie contrapposizioni) sono da registrare delle osmosi, ma fondamentalmente sul terreno concreto della prassi (mai della "dottrina") dei loro militanti laddove le circostanze storiche abbiano fatto prevalere, sulle divisioni, delle comuni esigenze di lotta: per esempio, sul terreno dell'opposizione al fascismo o nel fuoco vivo di rivolte generazionali come il Sessantotto. In altri termini, piuttosto che di vere e proprie osmosi si è trattato, come nei casi appena citati, di spontanee "convergenze d’impeto" delle rispettive basi di militanza determinate da un comune trascinamento negli impegni immediati e nelle passioni delle lotte. Quelle che chiamo "convergenze d'impeto" potevano essere registrate da parte degli avversari della sinistra nei termini di una pericolosa fusione di forze in un compatto fronte eversivo, ma chiunque conosca le cose dall'interno della sinistra sa bene che, a dispetto del comune trascinamento, $\mathrm{i}$

${ }^{22}$ Cfr. STAZZI, Carlo Andrea, «"And Now - Win the peace!”. I laburisti inglesi e il Welfare State», in Diacronie. Studi di Storia Contemporanea : Quando la classe operaia andava in paradiso, $\mathrm{N}^{\circ} 9,1 \mid 2012$,

URL: < http://www.studistorici.com/2012/02/13/stazzi_numero_9/ > . 
militanti dei vari gruppi mantenevano tenacemente le loro diverse mentalità e culture politiche. Il che è particolarmente vero per gli anarchici, ed anche con più evidenza per i cosiddetti "radicali", della cui reale appartenenza alla sinistra in senso proprio c'è sempre stato molto da dubitare, essendo essi in gran parte - quale che sia la loro spesso autentica autorappresentazione di "rivoluzionari" - nient'altro che l'estrema espressione giacobina del liberalismo (di per sé individualista e dalle indiscutibili origini "borghesi")23.

Ma veniamo ad un altro punto della domanda. Mi si chiede se ha ancora un senso la classica distinzione tra "riformisti" e "rivoluzionari". Ebbene, tutto quello che ho fin qui detto nel corso della nostra conversazione mi accredita come un fermo sostenitore della necessità, non solo teorica ma anche pratico-politica, di tener fede a tale distinzione. Lo spartiacque tra i due campi è costituito dall'orientamento nei confronti del sistema capitalistico. Occorre scegliere prendendo atto di una contraddizione non eludibile: un sistema fondato sul culto dell'individualismo e su organiche diseguaglianze nonché sul mito del cosiddetto "libero mercato" (imposto dall'egemonia capitalistica dopo il 1989) non è compatibile con una prospettiva di "democrazia reale" fondata sulla socializzazione e finalizzata alla giustizia sociale. Tertium non datur. Quindi, fare "opposizione" all'interno del sistema capitalistico con il fine ufficiale di "migliorarlo" è ben altra cosa di battersi per il suo superamento, a tal punto da far diventare persino dubbio che un'“opposizione” di tal genere sia da riconoscere come un'opposizione reale, appena sufficiente a definire un'identità di Sinistra (con la "S" maiuscola). Tra l'altro (ne ho già accennato) quanti riducono oggi l'essere di sinistra ad una vocazione "riformista" non sanno bene di che cosa stiano parlando: quali "riforme"? Riformare l'esistente per andare indietro? In Italia c'è già gente ufficialmente di sinistra (ma come dirla di Sinistra?) disposta ad accettare che siano da prendersi per "riforme" certe leggi che vanno in una direzione opposta alla socialità e alla socializzazione o che sono surrettiziamente delle "controriforme" come, per citarne una, quella della ministra Gelmini per l'Università. E, continuando su questa strada, potrebbero assumere come "riforma" anche un netto arretramento delle conquiste del mondo del lavoro accondiscendendo a modifiche regressive dell'art.18 dello Statuto dei lavoratori.

Certo, se proprio si volesse essere molto generosi con i concetti e con le parole, ci sarebbe da aver comprensione e rispetto per il fatto che siano esistiti, come continuano ad esistere, persino dei liberali (così come in passato dei "democristiani”) a loro modo decisi a ritenersi e a professarsi di "sinistra". Ma non credo che per "cambiare il mondo" qual è, e quale non vogliamo più che sia, risulti utile seguirli nelle loro

${ }_{23}$ Cfr. MARINO, Giuseppe Carlo, Le Generazioni italiane dall'Unità alla Repubblica, cit. 
presunzioni. Il che, mi sembra, è di particolare urgenza per una Sinistra che voglia riconquistarsi una sua certificante e non immaginaria identità di fronte al capitalismo globalizzato.

D.: Dal suo punto di vista, quali sono gli errori che la sinistra italiana ha compiuto nel trentennio 1945-1973? Quali sono stati i nodi non risolti che poi l'hanno portata alla crisi della fine degli anni Ottanta? Quali meriti e quale ruolo positivo hanno giocato le sinistre nel processo di ricostruzione materiale e morale in Italia dopo il ventennio fascista e la guerra?

G. C. M.: Con questa domanda, in pratica mi si chiede di ripercorrere l'intera storia politica e sociale della cosiddetta "prima repubblica". Francamente, non è un impegno che posso assumermi nei limiti di questa conversazione. Posso soltanto fissare alcuni punti fermi. E comincio con quelli che riguardano i "meriti" complessivi delle sinistre italiane: senza la loro azione, pur travagliata e spesso contraddittoria, non avremmo avuto la repubblica, la Costituzione di cui andiamo giustamente fieri, lo Stato sociale, lo Statuto dei lavoratori, la conquista di numerosi diritti civili per entrambi i sessi e, soprattutto, quel tanto che - nonostante i limiti e poi il recente degrado prodottosi sulla mentalità e sul costume di una larghissima area sociale con il berlusconismo - ci è dato di costatare in termini di capillare e diffusa educazione alla democrazia. E che dire quanto agli errori? A voler rispondere, gli interrogativi si moltiplicano. In che senso errori, se di errori è corretto parlare, e rispetto a chi e a che cosa? E poi, è meglio parlare di errori o di sbandamenti? E ancora: sbandamenti della prassi politica o concrete impossibilità di far diversamente nelle condizioni date di un Paese complesso e assai conflittuale come il nostro, con ceti popolari di recente e mai pienamente superata arretratezza, con un generalizzato costume tendente alla corruzione della vita pubblica e al trasformismo della politica? In ogni caso, errori, sbandamenti o impossibilità che siano, la loro identificazione e la loro valutazione dipendono dai due punti di vista, ben poco conciliabili tra loro, della sinistra socialdemocratica da una parte e della sinistra comunista e antagonista dall'altra. Per l'una, l'errore complessivo della sinistra sarebbe consistito nella sua mancata unificazione sul terreno del riformismo, con una Bad Godersberg italiana. E, con un siffatto giudizio - a parte molto improbabili valutazioni autocritiche circa l'inferiorità culturale e progettuale del loro miope ceto politico americanista rispetto alle ben più laica ed autonoma SPD tedesca - la responsabilità dell'errore verrebbe addossata alla controparte comunista e antagonista. Nodo irrisolto: la tardiva, e mai del tutto chiara, rottura del Pci con 
l'Unione sovietica; una mancata adesione al "mondo libero" e antitotalitario dell'Occidente antisovietico, indicata come responsabile della divisione delle forze socialiste, ovvero di una contrapposizione a sinistra determinata da due opposte e inconciliabili scelte di modelli di civiltà, alla quale la polemica politica ha fatto risalire, nella vita della repubblica, le cause di quellinterminabile stagione democristiana che, secondo Alberto Ronchey ${ }^{24}$ ed altri, avrebbe caratterizzato l'Italia come una "democrazia bloccata", incapace di sviluppare una dialettica democratica tra Sinistra e Destra in un rassicurante ambito liberaldemocratico: una democrazia sotto la vigilanza sempre allarmata degli americani (in sostanza in uno Stato consegnato ad una "sovranità limitata"), appiattitasi sul "centrismo" già strategicamente impostato da De Gasperi come unica formula politica possibile per l'Italia, mantenutasi negli anni, in un circolo vizioso di sostanziale stagnazione, a dispetto della timida e insufficiente variante del "centro-sinistra". Si sarebbe trattato, in altri termini, della mancata rimozione di quell'ostacolo all'unità della sinistra che Ronchey chiamava "fattore K", il fattore che più inquietava, e addirittura terrorizzava, le masse dei moderati italiani e che - in un orizzonte internazionale diviso nei due fronti avversi della guerra fredda - faceva gravare sulla Sinistra il divieto americano di accedere autonomamente alla guida del governo, dati gli sconvolgenti effetti che se ne temevano per il sistema di alleanze della Nato $^{25}$. Di contro, sia i comunisti che i più vari militanti della sinistra antagonista hanno abbondanti motivi per sostenere che soltanto il loro impegno di tenere in vita una strategia rivoluzionaria sulla linea strategica inaugurata dalla "rivoluzione di ottobre" (pur ancorata alla giudiziosa tattica della "guerra di posizione") ha consentito alla sinistra italiana di continuare ad esistere come... una vera Sinistra senza cedere alle sirene dell'americanismo. Semmai, nel caso specifico di questa sinistra rivoluzionaria, gli errori sarebbero da vedersi nelle non poche incertezze e incoerenze registratesi tra opportunismi e contaminazioni - penso, per le contaminazioni, a quel certo "migliorismo" formatosi come corrente all'interno del Pci - nel perseguimento di tale strategia, a partire dalla "doppiezza" già denunziata da Togliatti ${ }^{26}$.

24 Cfr. RONCHEY, Alberto, Accadde in Italia: 1968-1977, Milano, Garzanti, 1977; ID., Libro bianco sull'ultima generazione: tra candore e terrore, Milano, Garzanti, 1978.

${ }_{25}$ RONCHEY, Alberto, Chi vincerà in Italia? La democrazia bloccata, i comunisti e il fattore $K$, Milano, Mondadori, 1982.

${ }^{26}$ Cfr. AGOSTI, Aldo, Storia del Partito comunista italiano 1921-1991, Roma-Bari, Laterza, 1999; GALLI, Giorgio, Storia del PCI: Livorno 1921, Rimini 1991, Milano, Kaos edizioni, 1993; MARTINELli, Renzo, Storia del Partito Comunista Italiano, vol. 6, Il "partito nuovo" dalla Liberazione al 18 aprile, Torino, Einaudi, 1995; MARTINELLI, Renzo, GOZZINI, Giovanni, Storia del Partito Comunista Italiano, vol. 7, Dall'attentato a Togliatti all'VIII congresso, Torino, Einaudi, 1998; SPRIANO, Paolo, Storia del Partito Comunista Italiano, 5 voll., Torino, Einaudi, 1967-1975. 
Per il resto, mi tengo qui molto sulle generali per indicare qualcuno degli errori addebitabili quasi in esclusiva a quella parte della sinistra italiana che ha avuto modo di accedere in vari tempi al governo del Paese insieme alla Dc. Normalmente - se si fa un po' eccezione per il finale tentativo di Bettino Craxi di conquistarsi un ruolo più incisivo sfruttando la "rendita di posizione", ovvero di interdizione, assicuratagli dal suo oscillante e precario $10-14 \%$ in un parlamento formato con il sistema elettorale proporzionale - si trattò di un ruolo di governo segnato dalla subalternità. I socialisti nei governi Moro degli anni Sessanta e in quelli successivi del "centro-sinistra" si erano adattati, nonostante i loro programmi, a non ancorare gli sviluppi concreti dello "Stato sociale" ad un'organica "programmazione economica": il loro era stato, ben al di sotto di un vero e proprio socialismo, una specie di keynesismo d'abord ${ }^{27}$. Infine, negli anni ruggenti di Craxi e della "Milano da bere" vissuti nel connubio con la Dc di Andreotti (1983-1987, nonché i successivi al segno del vivace protagonismo craxiano), gli anni della corruzione sviluppatasi nel sistema di Tangentopoli, quei socialisti sempre meno connotati da credibile socialismo avrebbero interpretato il loro ruolo soprattutto come quello di una forza-guida della società dei consumi e del consumismo, favorendo la dilatazione delle spesa pubblica a scapito del bilancio dello Stato, il cui crescente indebitamento sarebbe stato scriteriatamente rinviato alle future generazioni. E, in tale allegro contesto, in uno con la stabilizzazione di un organico sistema di corruzione della politica e dell'economia, sarebbero diventati anche organici i rapporti con i poteri mafiosi più o meno occultamente radicatisi, in varie forme, nell'intero territorio nazionale, e resi ancora più forti da una contestuale espansione del fenomeno nel quadro internazionale. Il fatto che il prezzo dell'intera vicenda di dissesto e corruzione sia stato poi pagato soprattutto dal Psi (il più antico ed illustre, e per decenni benemerito, partito dei lavoratori italiani) con la sua fine ingloriosa proprio a cent'anni dalla sua fondazione, è un fatto che costituisce una sconfitta storica dell'intera sinistra italiana. Tanto pesante e tanto scottante quella sconfitta, da aver reso improponibile in Italia persino il nome "socialismo" 28 .

27 Cfr. VOULGARIS, Yannis, L'Italia del centro-sinistra 196o-1968, Roma, Carocci, 1998; COLARIZI, Simona et al., La cruna dell'ago. Craxi, il Partito Socialista e la crisi della Repubblica, Bari, Laterza, 2006. Sulla programmazione economica e la costruzione dello stato sociale in Italia si vedano DI FENIZIO, Ferdinando, La programmazione economica (19461962), Torino, Editrice Torinese, 1964; FORTE, Francesco, Introduzione alla politica economica. Il mercato e i piani, Torino, Einaudi, 1967; MINISTERO DEL BILANCIO, La programmazione economica in Italia, Roma, Società grafica, 1967; LOMBARDINI, Siro, La programmazione. Idee, Esperienze, Problemi, Torino, Einaudi, 1967.

${ }_{28}$ Si veda GALLI, Giorgio, Storia del socialismo italiano: da Turati al dopo Craxi, Milano, Baldini Castoldi Dalai editore, 2007; SPINI, Andrea (a cura di), Bettino Craxi, il riformismo e la 
D.: Quale ruolo ha giocato nella definizione di un'identità comune della sinistra europea la peculiarità della sinistra italiana, della sua storia così diversa da quella delle tradizioni europee - come quella socialista francese, quella socialdemocratica tedesca e scandinava, quella laburista inglese?

G. C. M.: Anche una risposta a questa domanda comporterebbe la fatica di scrivere, ben più che un saggio, un ampio e complesso trattato. Spero che qualcuno abbia mezzi e tempo per scriverlo. Da marxista, e comunista non pentito qual sono, ritengo che la sinistra italiana avrebbe potuto giocare (ma non ha giocato) un ruolo decisivo nella definizione di un'identità comune della sinistra europea, con Gramsci e con il gramscismo, ovvero con quella mirabile strategia elaborata da Gramsci per mettere in concreta sintonia democrazia e socialismo ai fini di un autentico cambiamento rivoluzionario nelle società avanzate dell'Occidente ${ }^{29}$. Ad assumere questo ruolo ci provò Enrico Berlinguer con la sua proposta di "eurocomunismo". Non ci riuscì. Può darsi, almeno lo spero, che qualche forza emergente della "sinistra che non si accontenta" prima o poi ci riesca, in forme nuove adeguate ai tempi. La lezione di Gramsci è sempre più attuale, così come sta riprendendo quota, dopo anni di oblio e di dileggiante abbandono, l'analisi marxista del capitalismo di fronte agli effetti devastanti del potere mondiale del capitalismo finanziario.

Detto questo, certo non va dimenticato il contributo rilevantissimo che una particolare sinistra italiana (quella liberalsocialista del partito d'azione nato dal lavoro politico e dalla tragica testimonianza di Carlo Rosselli) diede alla stessa idea di Europa, a partire dal Manifesto di Ventotene, scritto sotto la scure fascista, al confino di polizia, da due grandi esponenti della sinistra libertaria del livello di Altiero Spinelli e di Ernesto Rossi con proposte profetiche (poi, purtroppo, solo parzialmente valorizzate e largamente eluse dal processo di unificazione europea). Spostando l'attenzione alla sinistra socialdemocratico-migliorista, non è facile cogliere e vagliare un ruolo specifico svolto per la formazione di una sinistra europea dal Psi, al di là della costante recitazione "atlantista" ed americanista di un Giuseppe Saragat alla testa del suo gracile e corrotto Psdi $^{30}$. Si può immaginare - ma andrebbe specificamente studiato - il ruolo

sinistra italiana, Venezia, Marsilio, 2011; ID., Bettino Craxi, il socialismo europeo e il sistema internazionale, Venezia, Marsilio, 2006.

${ }_{29}$ Cfr, BOBBIO, Norberto, Saggi su Gramsci, Milano, Feltrinelli, 1990; MARTELLI, Michele, Gramsci filosofo della politica, Milano, Unicopli, 1996; LOSURDO, Domenico, Antonio Gramsci. Dal liberalismo al comunismo critico, Roma, Gamberetti editrice, 1997.

$3^{\circ}$ Cfr. ROSSELLI, Carlo, Socialismo liberale, Torino, Einaudi, 1973; ZUCARO, Domenico, «Il Quarto Stato» di Pietro Nenni e Rosselli, Milano, SugarCo, 1977; TERRACIANO, Nicola (a cura 
poi svolto da Bettino Craxi per lo sviluppo omogeneo, in Europa, di una sorta di spurio "socialismo liberista" o, se si preferisce, opportunista (da tempo Craxi aveva abbandonato Marx e il marxismo, inizialmente nel nome di un riscoperto Proudhon!) nel quadro delle intense relazioni intrattenute a fine secolo con i vari Tony Blair, Felipe Gonzéles, Francois Mitterand, Mario Soares, Michel Rocard e Andreas Papandreou.

\section{D.: Lei è un attento osservatore della realtà politica dell'Italia: come vede l'ormai più che decennale crisi d'identità della sinistra italiana e la difficile unità delle sue molte anime?}

G. C. M.: La crisi di identità della sinistra italiana (e non solo di quella italiana) è di lunga durata. Fa bene la domanda a suggerire che è "più che decennale". Sarebbe impossibile indicarne una precisa data di partenza. Si tratta, infatti, di un processo che è tutt'uno con gli sviluppi della trasformazione epocale (di cui ho già parlato) divenuta sempre più evidente, in Italia e in tutto l'Occidente, dagli anni Ottanta del secolo scorso in poi. Centrale in questa crisi, come ho già detto, è il portato delle profonde mutazioni indotte dalla "rivoluzione elettronico-informatica" nel sistema di produzione e nella morfologia delle classi e dei ceti sociali31․ La ricerca di identità va diventando affannosa, e forse ormai disperata, perché ci si accorge di non possederne una qualsiasi che risulti convincente. Per l'identità, accade esattamente quel che è dato constatare anche per la memoria storica: la si invoca tanto più ansiosamente, e con enfasi, quanto più ci si accorge che la si sta smarrendo. Il dato storico, in Italia, è che tutti i partiti dell'assai composita e frantumata sinistra sviluppatasi nellimperfetto processo di unificazione sociale di centocinquanta anni di Stato unitario non hanno varcato la soglia del nuovo millennio: né il Psi, né il Pci, né tutte le loro controverse e litigiose filiazioni e diaspore nel tempo. Ritengo che adesso la "forma-partito" (che è, come aveva già ben colto tempestivamente Pietro Ingrao, una forma politica ottocentesca, propria di una società di massa ancora appartenente al vecchio e superato quadro della "rivoluzione industriale") non sia la più idonea a dare organicità alla ricomposizione delle cosiddette "anime" della sinistra. Oltre tutto - a parte quel che serve ai fini della rappresentanza in parlamento tramite le dinamiche elettorali - non saprei proprio se una siffatta

di), Liberalismo socialista e socialismo liberale, Casalvelino Scalo, Galzerano Editore, 1992; LIMITI, Giuliana, DI NAPOLI, Mario (a cura di), Carlo e Nello Rosselli, Giustizia e libertà, Roma, UIL, 1993; DELL'ERBA, Nunzio, «Democrazia e socialismo in Giuseppe Saragat», in Tempo Presente, 6o, 1985 pp.39-45. Su Saragat cfr. anche DONNO, Michele, Giuseppe Saragat e il PSLI (1945-1952), Soveria Mannelli, Rubbettino, 2009.

${ }^{31}$ Cfr. RIFKIN, Jeremy, L'era dell'accesso. La rivoluzione della new economy, Milano, Mondadori, 2000. 
"organicità" sia ancora necessario proporsela come un traguardo. Il futuro probabilmente si costruisce nell'attivazione (tramite canali tra i quali Internet sarà sempre più chiamato a svolgere un ruolo fondamentale) di ampie aree di dibattito collettivo e di dialettici movimenti interindividuali di critica all'esistente e di consequenziale impegno politico. Naturalmente per una siffatta attivazione e per la l'espansione sociale del processo saranno ancora importanti i gruppi di "militanza", con un ruolo analogo, seppure ampiamente rinnovato, a quello che Lenin e Gramsci attribuivano alle "avanguardie".

\section{D.: E in chiave europea di quale identità possiamo parlare oggi per la sinistra? Il riformismo è l'unico destino possibile per la sinistra europea?}

G. C. M.: Se tentassi di dare una risposta soltanto per rispondere, mi sentirei colpevolmente elusivo e imperdonabilmente retorico. Quanto ho già detto per l'Italia mi sembra valga anche per l'Europa. Aggiungo, per dirla con l'acuto Zygmunt Bauman, che in una "società liquida" è normale che diventino altrettanto "liquide", anche le idee e le culture politiche ${ }^{32}$. La cosa, ovviamente, vale sia per quel che si è ancora soliti indicare come sinistra, sia per quel che, di contro, si indica (forse con minore pericolo di sbagliarne l'identificazione) come destra. Rimane certo, al di là della loro "liquefazione" (o, se si preferisce della loro consunzione), che le due parole continuano ad assolvere un ruolo identitario, e a mantenere un valore significante, proprio attraverso la loro contrapposizione: si è di "sinistra" perché non si è di "destra" e viceversa. Certamente sono parole da usare in un modo diverso rispetto all'uso che se ne faceva in passato, dati i profondi cambiamenti strutturali che la grande trasformazione in corso (la "rivoluzione della postmodernità) ha determinato a carico dei loro rispettivi, tradizionali soggetti sociali di riferimento: il proletariato industriale (la classe operaia) e il padronato (la borghesia nazionale). Tuttavia, il doverle usare adesso in modo diverso, non fa affatto venir meno per una sinistra che voglia ancora così "identificarsi”, il suo compito storico di lottare contro tutte le forze manifeste o surrettizie della "destra" che siano di ostacolo alla realizzazione di una "democrazia reale" (di per sé necessariamente anticapitalistica), idonea a rimuovere le ingiustizie e a conseguire il più ampio rispetto dei diritti umani in una nuova società il più possibile egualitaria. Se, nella condizioni imposte dalla "postmodernità", tutto questo appare come un'utopia, ebbene - l'ho già detto - è giocoforza per la sinistra attivare una

32 BAUMAN, Zygmunt, Modernità liquida), Roma-Bari, Laterza, 2002. 
dialettica tra l'utopia e i processi reali della trasformazione. Mi sembra che dal mondo giovanile stiano emergendo dei segnali incoraggianti, in una siffatta direzione. Il laboratorio per la formazione di una nuova sinistra è appena aperto e non è dato prevedere a quali risultati perverrà in futuro una difficile elaborazione che non può comunque dipendere né da tenere nostalgie per un grande e nobile passato ormai irrimediabilmente archiviato, né da interminabili tentativi di "rifondazione" di travolte esperienze. Meno che mai, da un adeguamento, che pur si reputi giudizioso e voglia proporsi con animo "progressista", ai diktat della globalizzazione capitalistica. Se il cosiddetto "riformismo" (che è appunto, un siffatto adeguamento subalterno, per quanto a volte sofferto) fosse l'unico destino della sinistra europea, dovremmo rassegnarci in Europa a non avere più una... Sinistra! Nessuno, infatti, neppure con la migliore finesse di valutazione, riuscirebbe più a distinguerla dalla Destra. 


\section{* L'autore}

Giuseppe Carlo Marino è professore ordinario di Storia Contemporanea nella Facoltà di Scienze politiche dell'Università di Palermo. Studioso dei rapporti tra potere e società in Italia, collaboratore della RAI impegnato sul fronte dell'uso pubblico della storia, è autore di numerosi libri, tra i quali L'Opposizione mafiosa, Palermo, Sellerio, 1964; La formazione dello spirito borghese in Italia, Firenze, La Nuova Italia, 1974; Storia del separatismo siciliano, Roma, Editori Riuniti, 1976; L'autarchia della cultura, Roma, Editori Riuniti, 1983; Autoritratto del Pci staliniano, Roma, Editori Riuniti, 1991; La repubblica della forza, Milano, Franco Angeli, 1996; Storia della mafia, Roma, Newton \& Compton, 1998; Eclissi del principe e crisi della storia, Milano, Franco Angeli, 2000; Padrini, Roma, Newton \& Compton, 2001; Biografia del Sessantotto, Milano, Bompiani, 2004.

URL: < http://www.studistorici.com/progett/comitato-scientifico/ >

\section{Per citare questo articolo:}

BUFARALE, Luca, PIETRANCOSTA, Fausto, «Intervista a Giuseppe Carlo Marino», Diacronie. Studi di Storia Contemporanea: Quando la classe operaia andava in paradiso, 13/2/2012,

URL:<http://www.studistorici.com/2012/02/13/marino_numero_9/ >

\section{Diacronie Studi di Storia Contemporanea $\beta$ www.diacronie.it}

Risorsa digitale indipendente a carattere storiografico. Uscita trimestrale. redazione.diacronie@hotmail.it

Comitato di redazione: Marco Abram - Giampaolo Amodei - Jacopo Bassi - Luca Bufarale - Alessandro Cattunar - Alice De Rensis Barbara Galimberti - Deborah Paci - Fausto Pietrancosta - Martina Sanna - Matteo Tomasoni - Luca Zuccolo

Diritti: gli articoli di Diacronie. Studi di Storia Contemporanea sono pubblicati sotto licenza Creative Commons 2.5. Possono essere riprodotti a patto di non modificarne i contenuti e di non usarli per fini commerciali. La citazione di estratti è comunque sempre autorizzata, nei limiti previsti dalla legge. 